\title{
On vector spaces of certain modular forms of given weights
}

\section{A.R. Aggarwal and M.K. Agrawal}

Let $p$ be a rational prime and $Q_{p}$ be the field of $p$-adic numbers. Jean-Pierre Serre [Lecture Notes in Mathematics, 350 , 191-268 (1973) ] had defined p-adic modular forms as the limits of sequences of modular forms over the modular group $\mathrm{SL}_{2}(Z)$. He proved that with each non-zero p-adic modular form there is associated a unique element called its weight $k$. The p-adic modular forms having the same weight form a $Q_{p}$-vector space. The object of this paper is to obtain a basis of p-adic modular forms and thus to know precisely all p-adic modular forms of a given weight $k$. The dimension of such modular forms as a $Q_{p}$ vector space is countably infinite.

\section{Notations and definitions}

Let $Z$ be the ring of rational integers and $Q$ the field of rational numbers. From now on we will write for the modular forms over $\mathrm{SL}_{2}(Z)$ simply "modular forms".

Let $\nu_{p}$ denote the valuation of the field of p-adic numbers $Q_{p}$ which is normalised so that $v_{p}(p)=1$. Let $Z_{p}$ be the ring of $p$-adic integers; that is, $Z_{p}=\left\{x \mid x \in Q_{p}, \nu_{p}(x) \geq 0\right\}$.

For an even integer $k \geq 2$, take

Received 13 January 1977. 


$$
E_{k}=1-\frac{2 k}{b_{k}} \sum_{n=1}^{\infty} \sigma_{k-1}(n) q^{n}
$$

where $b_{k}$ is the $k$ th Bernoulli number and $\sigma_{k-1}(n)=\sum_{d\rceil n} d^{k-1}$. For $k \geq 4, E_{k}$ is a modular form of weight $k$. We will denote $E_{2}, E_{4}$, and $E_{6}$ by $P, Q, R$ respectively.

As usual, we take,

$$
\Delta=2^{-6} 3^{-3}\left(Q^{3}-R^{2}\right)=\sum_{n=1}^{\infty} \tau(n) q^{n}=q \prod_{1}^{\infty}\left(1-q^{n}\right)^{24}
$$

Then $\Delta$ is a cusp form of weight 12 .

Let $Q_{p}[[q]]$ be the ring of formal power series in $q$ with coefficients in $Q_{p}$.

DEFINITION 1.1. Let $f=\sum_{n=0}^{\infty} a_{n} q^{n} \in Q_{p}[[q]]$. Define

$$
v_{p}(f)=\inf _{n} v_{p}\left(a_{n}\right)
$$

DEFINITION 1.2. Let $\left\{f_{i}\right\}$ be a sequence of elements of $Q_{p}[[q]]$. We say that $f_{i} \rightarrow f$, if the coefficients of $f_{i}$ tend uniformly to those of $f$; that is, $\nu_{p}\left(f-f_{i}\right) \rightarrow \infty$ as $i \rightarrow \infty$.

DEFINITION 1.3. Let $\left\{f_{i}\right\}, f_{i}=\sum_{n=0}^{\infty} a_{n}^{(i)} q^{n}$, be a sequence of modular form with coefficients $a_{n}^{(i)}$ rational. Let $f_{i} \rightarrow f=\sum_{n=0}^{\infty} a_{n} q^{n}$, with $a_{n}$ in $Q_{p}$, in the sense of Definition 1.2. Then $f$ is called a p-adic modular form.

DEFINITION 1.4. Let $m$ be an integer greater than or equal to $I$ if $p \neq 2$ and $m \geq 2$ if $p=2$. Define 


$$
\begin{aligned}
& \text { Vector spaces of modular forms } \\
& X_{m}= \begin{cases}\left(Z / p^{m-1} Z\right) \times Z /(p-1) Z, & \text { if } p \neq 2, \\
Z / 2^{m-2} Z, & \text { if } p=2 .\end{cases}
\end{aligned}
$$

Let $X$ be the limit of the projective system $\left\{x_{m}\right\}$. Then

$$
X=\underset{\lim }{\longleftarrow} X_{m}= \begin{cases}z_{p} \times Z /(p-1) Z, & \text { if } p \neq 2, \\ Z_{2}, & \text { if } p=2 .\end{cases}
$$

Now we have the following theorem proved in [1].

THEOREM (Serre). Let $f$ be a p-adic modular form and let $\left\{f_{i}\right\}$ be a sequence of modular forms with rational coefficients. Let the weight of $f_{i}$ be $k_{i}$ and let $f_{i} \rightarrow f$. Then $\left\{k_{i}\right\}$ has a limit $k$ in the group $X$. This limit depends only on $f$ and not on the choice of the sequence $\left\{f_{i}\right\}$

DEFINITION 1.5. The limit $k$ in $X$ of $\left\{k_{i}\right\}$ as stated in the above theorem of Serre is called the weight of the p-adic modular form f.

DEFINITION 1.6. A series $\sum_{n=0}^{\infty} g_{n}$, where the $g_{n}$ 's are $p$-adic modular forms is called an isobaric series if each $g_{n}$ has the same weight.

REMARK 1. In the theorem of Serre, since each $k_{i}$ is an even integer, the limit $k$ of $\left\{k_{i}\right\}$ is an element of $2 X$. This implies that

$$
\begin{aligned}
& \text { (i) if } p \text { is an odd prime and } k=(s, u) \text {, then } s \in Z_{p} \text { and } \\
& u \text { is an even integer } \bmod (p-1) \text {, and } \\
& \text { (ii) if } p=2 \text {, then } k=s \text {, with } s \in Z_{2} \text {. }
\end{aligned}
$$

REMARK 2. If $\left\{f_{i}\right\}$ is a sequence of p-adic modular forms with 
weights $k_{i}$, and $f_{i} \rightarrow f$ then $f$ is a p-adic modular form. We prove the following elementary lemma.

LEMMA. Let $f$ and $g$ be any two formal series in $q$ with coefficients from $Q_{p}$. Then,

$$
v_{p}(f g)=v_{p}(f)+v_{p}(g)
$$

Proof. Let

$$
f=\sum_{n=0}^{\infty} a_{n} q^{n}
$$

and

$$
g=\sum_{n=0}^{\infty} b_{n} q^{n}
$$

where $a_{n}, b_{n} \in Q_{p}$. Also let $\nu_{p}(f)=A$ and $\nu_{p}(g)=B$. Let

$$
\begin{aligned}
& \text { (i) } v_{p}\left(a_{k}\right)=A, v_{p}\left(a_{n}\right)>A \text { for } n=0,1, \ldots, k-1 \text {, and } \\
& \text { (ii) } v_{p}\left(b_{l}\right)=B, v_{p}\left(b_{n}\right)>B \text { for } n=0,1, \ldots, 2-1 .
\end{aligned}
$$

Now $f g=\sum_{n=0}^{\infty} e^{n} q^{n}$, where $c_{n}=\sum_{i+j=n} a_{i} b_{j}$. So

$\nu_{p}(f g)=\inf _{n} \nu_{p}\left(c_{n}\right) \geq A+B$. Also $\nu_{p}\left(c_{k+l}\right)=\nu_{p}\left(\sum_{i+j=k+l} a_{i} b_{j}\right)=A+B$,

whence $\nu_{p}(f g) \leq A+B$. Combining the two inequalities, we obtain the desired result. $/ /$

We can imbed $Z_{p}$ in $X$ if $p \neq 2$ by mapping $s \in Z_{p}$ to $(s, 0)$ in $X$. Now we prove the following theorem.

THEOREM 1. Let $p$ be a prime number greater than or equal to 5 and let $s \in Z_{p}$. Let $\left\{s_{n}\right\}$ be a sequence of non-negative rational integers such that $s_{n} \rightarrow s$ in $Z_{p}$. Then the sequence $\left\{\begin{array}{c}s_{n} \\ p-1\end{array}\right\}$ of modular forms (the weight of $E_{p-1}^{s}$ is $(p-1) s_{n}$ ), is convergent in the sense of Definition 1.2 and its limit is a p-adic modular form of weight ( $p-1) s$. 
Proof. Since $s_{n} \rightarrow s$ in $Z_{p}$, so $s_{n+1} \equiv s_{n} \bmod p^{n+1}$ for $n=0,1,2, \ldots$. Let $\left|s_{n+1} s_{n}\right|=\lambda_{n+1} p^{n+1}$, where $\lambda_{n+1}$ is an integer greater than or equal to 0 . Hence

$$
{ }_{E_{p-1}^{n+1}}^{s_{n+1}}-E_{p-1}^{s}{ }_{n-1}^{t}=E_{p-1}^{n}\left(E_{p-1}^{\lambda_{n+1} p^{n+1}}-1\right) \text {, }
$$

where $t_{n}=\min \left(s_{n+1}, s_{n}\right)$, and $\varepsilon=1$ or -1 .

Therefore

$$
\begin{aligned}
\nu_{p}\left(\begin{array}{c}
E_{p-1}^{n+1}-E^{s}{ }_{p-1}^{n} \\
p-1
\end{array}\right. & =\nu_{p}\left(E_{p-1}^{\lambda_{n+1} p^{n+1}}-1\right) \text { by Lemma } 1 \text { and the fact that } \nu_{p}\left(E_{p-1}\right)=0 \\
& \geq(n+1) .
\end{aligned}
$$

Thus $\left\{\begin{array}{c}s \\ E \\ p-1\end{array}\right\}$ is a convergent sequence of modular forms. Let its limit be denoted by $E_{p-1}^{s}$ in the sense of Definition 1.2. Hence $E_{p-1}^{s}$ is a $p$-adic modular form and its weight is $\lim _{n \rightarrow \infty}(p-1) s_{n}=(p-I) s$.

REMARK. The case $p=2,3$. If we take $E_{2}$ in place of $E_{p-1}$ and replace the word "modular forms" by p-adic modular forms in the above theorem then by using Corollaire 2 of Theorème 21' of Serre [1] we find the theorem holds in these cases too.

Let $p$ be an odd prime and let $f$ be any $p$-adic modular form of weight $k=(s, u), s \in Z_{p}, 0 \leq u<p-1$, and $u$ is even for $n=0,1,2, \ldots$. Choose any non-negative integers $a(n)$ and $b(n)$ satisfying

$$
4 a(n)+6 b(n)+12 n \equiv u \bmod (p-1) .
$$

Consider

$$
f_{n}=Q^{a(n)}{ }_{R}^{b(n)} \Delta^{n} E_{p-1}^{s}
$$

for $n=0,1,2, \ldots$, where

$$
s_{n}=\frac{s-[12 n+4 a(n)+6 b(n)]}{p-1} \in Z_{p}
$$


From Theorem 1, it follows that $E_{p-1}^{s} n$ is a p-adic modular form of weight $(p-1) s_{n}$ and hence $f_{n}$ is a p-adic modular form of weight $k=(\varepsilon, u)$.

Let

$$
f_{n}=\sum_{m=0}^{\infty} a_{m}^{(n)} q^{m}
$$

Since $\Delta^{n}=q^{n}+\ldots$, and the constant term in each of $Q, R$, and $E_{p-1}$ is 1 , so

$$
a_{m}^{(n)}=0 \text { for } 0 \leq m<n \text { and } a_{n}^{(n)}=1
$$

With the notations as above for $f_{n}$ we have the following theorem.

THEOREM 2. $f$ is a p-adic modular form of weight $k$ iff $f=\sum a_{n} f_{n}$ with $v_{p}\left(a_{n}\right) \rightarrow \infty$ as $n \rightarrow \infty$.

Proof. Let $f$ be a $p$-adic modular form and let

$$
f=\sum b_{m}^{(0)} q^{m}, b_{m}^{(0)} \in Q_{p} .
$$

The series $f-b_{0}^{(0)} f_{0}$ has no constant term and is a p-adic modular form of weight $k$. Let

$$
f-b_{0}^{(0)} f_{0}=\sum_{m=1}^{\infty} b_{m}^{(1)} q^{m}
$$

Now consider

$$
f-b_{0}^{(0)} f_{0}-b_{1}^{(1)} f_{1}\left(=\sum_{m=2} b_{m}^{(2)} q^{m}(\text { say })\right) .
$$

It is a p-adic modular form of weight $k$. Continuing this process, we see that $f-\sum_{\gamma=0}^{t} b_{\gamma}^{(\gamma)} f_{\gamma}$ is a p-adic modular form of weight $k$ for every non-negative integer $t$, and has no terms containing $q^{m}$ for $m=0,1, \ldots, t$. Hence we can find $b_{\gamma}^{(\gamma)} \in Q_{p}(\gamma=0,1,2, \ldots)$ such 
that as formal series in $q, f=\sum_{n=0}^{\infty} b_{n}^{(n)} f_{n}$; that is, $f=$ lim $g_{t}$ where $g_{t}=\sum_{\gamma=0}^{t} b_{\gamma}^{(\gamma)} f_{\gamma}$. Now each $g_{t}$ is a p-adic modular form of weight $k$ and $\left\{g_{t}\right\}$ is a convergent sequence, so $\nu_{p}\left(g_{t+1}-g_{t}\right)+\infty$ as $t \rightarrow \infty$. Hence $v_{p}\left(b_{t+1}^{(t+1)}-f_{t+1}\right) \rightarrow \infty$ with $t$. Now $v_{p}\left(f_{t+1}\right)=0$ for each $t$, so $v_{p}\left(b_{t+1}^{(t+1)}\right) \rightarrow \infty$. Hence taking $b_{n}^{(n)}=a_{n}$, we get $f=\sum a_{n} f_{n}$ with $\nu_{p}\left(a_{n}\right) \rightarrow \infty$ as $n \rightarrow \infty$.

Conversely let $f=\sum_{n=0}^{\infty} a_{n} f_{n}$, with $\left(a_{n}\right) \rightarrow \infty$ as $n \rightarrow \infty$. As above, taking $g_{t}=\sum_{\gamma=0}^{t} a_{\gamma} f_{\gamma}$, which is a p-adic modular form of weight $k$. Since $\nu_{p}\left(a_{n}\right) \rightarrow \infty$ with $n$ and $\nu_{p}\left(f_{n}\right)=0$, so $\left\{g_{t}\right\}$ is a convergent sequence with its limit equal to $f$. Hence $f$ is a p-adic modular form.

COROLLARY 1. Any p-adic modular form can be written as an isobaric series in $Q, R$, and $E_{p-1}$.

Proof. Obvious.

COROLLARY 2. The dimension of the $Q_{p}$-vector space of p-adic modular forms of weight $k$ is countably infinite.

Proof. In view of property (3) above the $f_{n}^{\prime}$ 's are linearly independent over $Q_{p}$. Also from Theorem 2 any $p$-adic modular form can be written as a linear combination over $Q_{p}$ of $f_{n}^{\prime} s$. So $\left\{f_{n} \mid n=0,1,2, \ldots\right\}$ is a basis of $p$-adic modular forms of the given weight $k$.

REMARK 1. The case $p=2$. Here we take

$$
f_{n}=\Delta^{n} E_{2}^{B} n, s_{n}=\frac{8-12 n}{2}
$$

Since $s \in 2 Z_{2}$, so $s_{n} \in Z_{2}$. These $f_{n}^{\prime}$ 's have the property (3) and 
Theorem 2 and its corollaries are true if we replace $E_{p-1}$ by $E_{2}(=P)$.

REMARK 2. With the notations of [1],

$\left\{\Delta^{n} E_{k-12 n}^{*} \mid n=0,1,2,3, \ldots\right\}$ also forms a basis of $p$-adic modular forms of weight $k$.

\section{Reference}

[1] Jean-Pierre Serre, "Formes modulaires et fonctions zêta p-adiques", Moduzar functions of one variable III, 191-268 (Proc. Internat. Summer School, University of Antwerp, RUCA, 1972. Lecture Notes in Mathematics, 350. Springer-Verlag, Berlin, Heidelberg, New York, 1973).

\footnotetext{
Department of Mathematics,

Panjab University,

Chandigarh,

India,

and

I.B. College,

Panipat,

India;

Department of Mathematics,

Panjab University,

Chandigarh,

India.
} 